\title{
Azithromycin for sarcoidosis cough: an open-label exploratory clinical trial
}

\author{
Simon D. Fraser ${ }^{1}$, Susannah Thackray-Nocera ${ }^{2}$, Marica Shepherd ${ }^{2}$, \\ Rachel Flockton ${ }^{2}$, Caroline Wright ${ }^{1,2}$, Wayne Sheedy ${ }^{1}$, Kayleigh Brindle ${ }^{2}$, \\ Alyn H. Morice ${ }^{1}$, Paul M. Kaye ${ }^{3}$, Michael G. Crooks ${ }^{1}$ and Simon P. Hart ${ }^{1}$
}

Affiliations: ${ }^{1}$ Respiratory Research Group, Hull York Medical School, Castle Hill Hospital, Cottingham, UK. ${ }^{2}$ Respiratory Clinical Trials Unit, Hull University Teaching Hospitals NHS Trust, Castle Hill Hospital, Cottingham, UK. ${ }^{3}$ York Biomedical Research Institute, University of York, York, UK.

Correspondence: Simon P. Hart, Respiratory Research Group, Hull York Medical School, Castle Hill Hospital, Cottingham, HU16 5JQ, UK. E-mail: s.hartRhull.ac.uk

\section{ABSTRACT}

Background: Chronic cough is a distressing symptom for many people with pulmonary sarcoidosis. Continuous treatment with a macrolide antibiotic may improve cough. We aimed to assess the potential efficacy of azithromycin in patients with sarcoidosis and self-reported cough.

Methods: We conducted a noncontrolled, open-label clinical trial of azithromycin $250 \mathrm{mg}$ once daily for 3 months in patients with pulmonary sarcoidosis who reported a chronic cough. The primary outcome was number of coughs in $24 \mathrm{~h}$. Secondary outcomes were cough visual analogue scales and quality of life measured using the Leicester Cough Questionnaire and King's Sarcoidosis Questionnaire. Safety outcomes included QTc interval on ECG. Measurements were made at baseline and after 1 and 3 months of treatment.

Results: All 21 patients were white, median age 57 years, 9 males, 12 females, median 3 years since diagnosis. Five were taking oral corticosteroids and none were taking other immunosuppressants. Twenty patients completed the trial. The median (range) number of coughs in $24 \mathrm{~h}$ was 228 (43-1950) at baseline, $122(20-704)$ at 1 month, and $81(16-414)$ at 3 months $(p=0.002$, Friedman's test). The median reduction in cough count at 3 months was $49.6 \%$. There were improvements in all patient-reported outcomes. Azithromycin was well tolerated.

Conclusion: In a noncontrolled open-label trial in people with sarcoidosis who reported a chronic cough, 3 months of treatment with azithromycin led to improvements in a range of cough metrics. Azithromycin should be tested as a treatment for sarcoidosis cough in a randomised placebo-controlled trial.

@ERSpublications

In a noncontrolled open-label trial in people with sarcoidosis who reported a chronic cough, 3 months of treatment with azithromycin led to improvements in a range of cough metrics https://bit.ly/2FB5tfq

Cite this article as: Fraser SD, Thackray-Nocera S, Shepherd M, et al. Azithromycin for sarcoidosis cough: an open-label exploratory clinical trial. ERJ Open Res 2020; 6: 00534-2020 [https://doi.org/ $10.1183 / 23120541.00534-2020]$.

This study is registered with the EU Clinical Trials Register (EudraCT 2019-000580-24). Individual participant data will not be shared.

Received: 28 July 2020 | Accepted after revision: 9 Sept 2020

Copyright $\odot$ ERS 2020. This article is open access and distributed under the terms of the Creative Commons Attribution Non-Commercial Licence 4.0. 


\section{Introduction}

People with sarcoidosis need treatment options that improve symptoms and quality of life, without causing undesirable side effects. Chronic cough is a distressing symptom for many patients with pulmonary sarcoidosis $[1,2]$. Continuous treatment with the macrolide antibiotic azithromycin may improve cough, although strong evidence is lacking [3,4]. Azithromycin is a cheap, readily available generic drug. In clinical trials in patients with chronic airways disease, continuous treatment with azithromycin for up to 1 year reduced exacerbations and was safe and well tolerated [4]. Whether azithromycin will benefit patients with sarcoidosis can only be answered definitively by a large randomised controlled trial (RCT). We conducted a single-centre open-label noncontrolled clinical trial of azithromycin in patients with pulmonary sarcoidosis to estimate the effect size and inform design of a future RCT.

\section{Methods}

The regional ethics committee (19/YH/0100) granted approval. The trial was registered on the European Union Drug Regulating Authorities Clinical Trials Database (EudraCT 2019-000580-24). Patients were recruited from the sarcoidosis clinic at Hull University Teaching Hospitals NHS Trust. Eligibility criteria were a diagnosis of pulmonary sarcoidosis, $>6$ months from diagnosis without evidence of self-resolving disease and self-reported chronic cough attributed to sarcoidosis. Patients could be taking a stable dose of prednisolone and/or other immunomodulatory drugs. The main exclusion criteria were current long-term macrolide therapy, allergy or intolerance to macrolide antibiotics, QTc prolongation on ECG (males $>450 \mathrm{~ms}$, females $>470 \mathrm{~ms}$ ), history of clinically significant cardiac arrhythmias, severe liver or kidney disease or clinically meaningful bronchiectasis. All patients received oral azithromycin $250 \mathrm{mg}$ once daily for 3 months. There was no placebo group.

Assessments were performed at baseline and at 1 month and 3 months on azithromycin therapy. The primary end-point was home-based 24-h cough counting using the Hull automated cough counter with the Leicester software algorithm [5]. Secondary end-points were visual analogue scale ((VAS) 0-100 mm) for cough severity and urge to cough, and quality of life assessments relating to cough and sarcoidosis using the Leicester cough questionnaire (LCQ) and King's sarcoidosis questionnaire (KSQ), respectively. KSQ scores for general health status (GH), lung and combined lung_GH modules were calculated following transformation of raw scores to a linear logit scale [6]. This was an exploratory study designed to inform a future RCT and no sample size calculation was performed. Statistical analyses were performed using IBM SPSS v.26. No imputation of missing data was performed and only participants with complete data were included in the repeated-measures analyses. Subgroup analyses of cough counts were performed using baseline cough severity VAS $\geqslant 40 \mathrm{~mm}$ to stratify participants, as this is common practice in chronic cough clinical trials.

\section{Results}

Details of the 21 participants are shown in table 1 . Their median age was 57 years, comprising 9 men and 12 women. All patients were white, reflecting the local population. The consort diagram is shown in figure 1 . Twenty participants completed the trial.

\section{TABLE 1 Demographic and clinical details of 21 recruited patients with sarcoidosis}

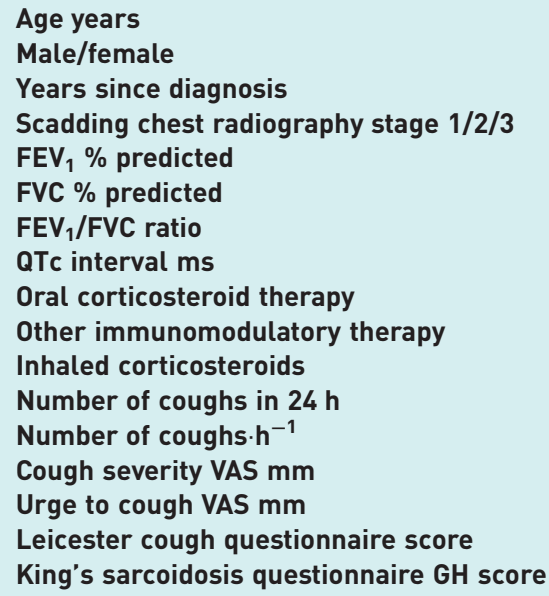

$57(48-71)$

$9 / 12$

$3(1-13)$

$2 / 6 / 13$

$87.5(52-131)$

$91.5(63-128)$

$0.75(0.55-0.93)$

$427(370-463)$

5

0

3

$228(43-1950)$

$10(2-81)$

$31(9-94)$

$26(8-94)$

$15.96(5.07-19.74)$

$50.7(23.8-100)$

Data are presented as median (range) or $\mathrm{n} . \mathrm{FEV}_{1}$ : forced expiratory volume in $1 \mathrm{~s}$; FVC: forced vital capacity; VAS: visual analogue scale. 


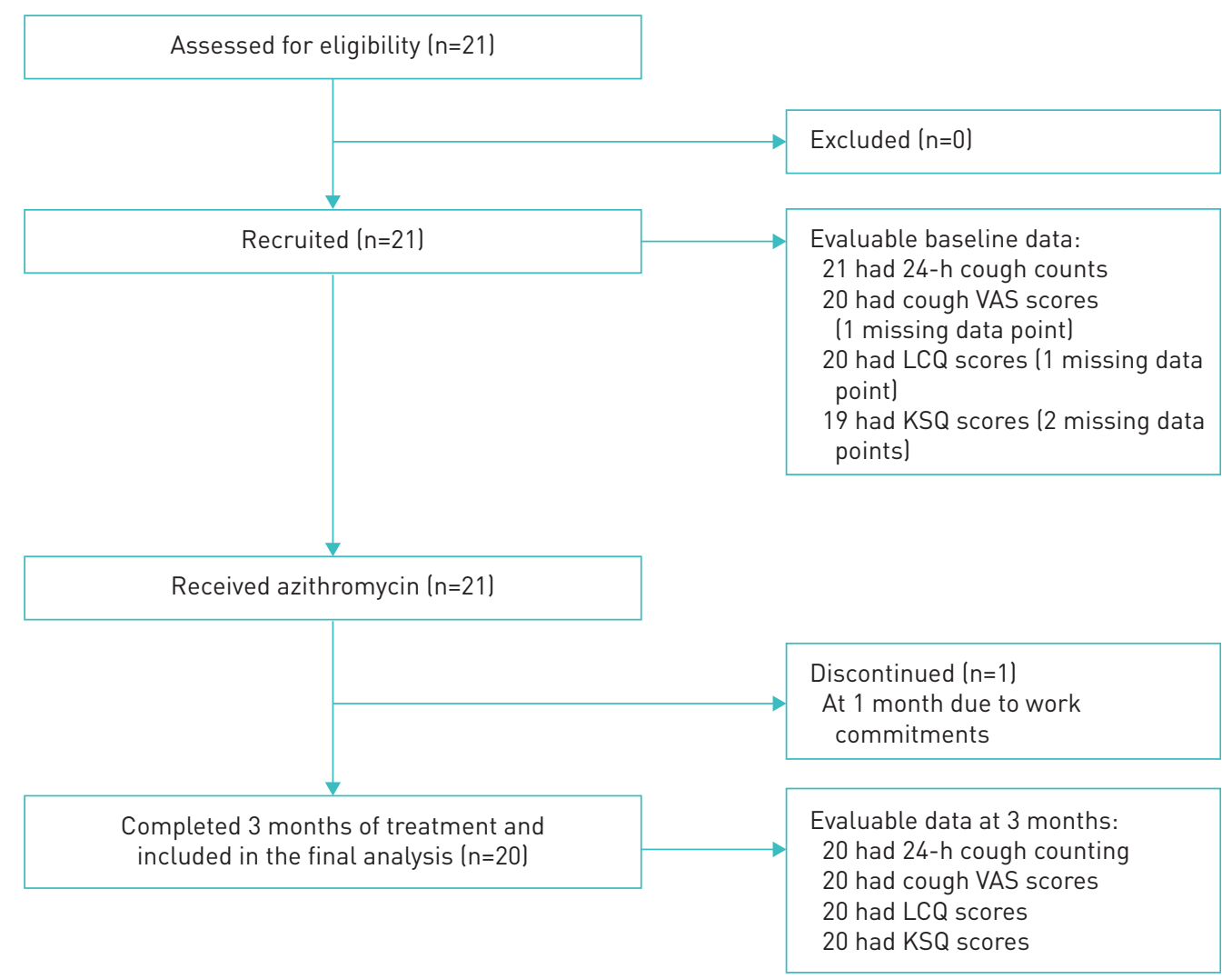

FIGURE 1 CONSORT diagram. VAS: visual analogue scale; LCQ: Leicester cough questionnaire; KSQ: King's sarcoidosis questionnaire.

The median (range) number of coughs in $24 \mathrm{~h}$ was 228 (43-1950) at baseline, $122(20-704)$ at 1 month, and $81(16-414)$ at 3 months ( $p=0.002$, Friedman's nonparametric ANOVA) (figure 2). After 3 months of azithromycin therapy, the median percentage reduction in the number of coughs was $49.6 \%$. To exclude the possibility that corticosteroid therapy affected cough responses, a sensitivity analysis of participants who were not taking oral steroids was performed which showed a similar result to the whole population (data not shown).

There were improvements in cough severity VAS, urge to cough VAS, LCQ scores, and KSQ scores (figure 3 and table 2). For 19 participants with complete data at 3 months, 11 (58\%) had clinically meaningful improvements in LCQ score (minimum clinically important difference (MCID) $\geqslant 1.3$ points [7]). Considering meaningful improvements in the KSQ [8], 15 participants (79\%) had an increase in KSQ_GH score of $>8$ points, $12(63.2 \%)$ had an increase in KSQ_lung score of $>4$ points and $13(68.4 \%)$ had an increase in KSQ_lung_GH score of $>5$ points. Absolute changes in cough counts correlated with changes in LCQ ( $\mathrm{r}=-0.637, \mathrm{r}^{2}=0.41, \mathrm{p}=0.003$, Pearson's correlation) and KSQ_GH $\left(\mathrm{r}=-0.587, \mathrm{r}^{2}=0.34\right.$, $\mathrm{p}=0.008$ ), but not with KSQ_lung or KSQ_lung_GH scores.

We looked at the impact of baseline cough severity VAS scores on cough count and response to azithromycin therapy. Baseline cough count was significantly higher in patients with baseline cough severity VAS $>40 \mathrm{~mm}$ compared with $<40 \mathrm{~mm}$ (median 365 versus 125 coughs in $24 \mathrm{~h}, \mathrm{p}=0.001$ (Mann-Whitney U-test)). After 3 months of azithromycin therapy, the median percentage reduction in cough count was $-78.8 \%$ in patients with baseline VAS $>40 \mathrm{~mm}$ compared with $-40.6 \%$ in patients with baseline $\mathrm{VAS}<40 \mathrm{~mm}(\mathrm{p}=0.057)$.

Ten participants reported 16 adverse events, 11 mild and 5 moderate, one of which led to temporary interruption of azithromycin therapy. No participant had to stop treatment permanently because of adverse events. The most common adverse event was the common cold in six participants (the trial was conducted over the winter), with two of these reporting transient worsening cough. Two participants reported transient gastrointestinal symptoms (stomach cramps, slight reduction in appetite). There were no serious adverse events. The median QTc interval on 12-lead ECG was $427 \mathrm{~ms}$ (range 370-463) at baseline, $428 \mathrm{~ms}(389-461)$ at 1 month and $435 \mathrm{~ms}(397-468)$ at 3 months $(\mathrm{p}=0.214)$. 


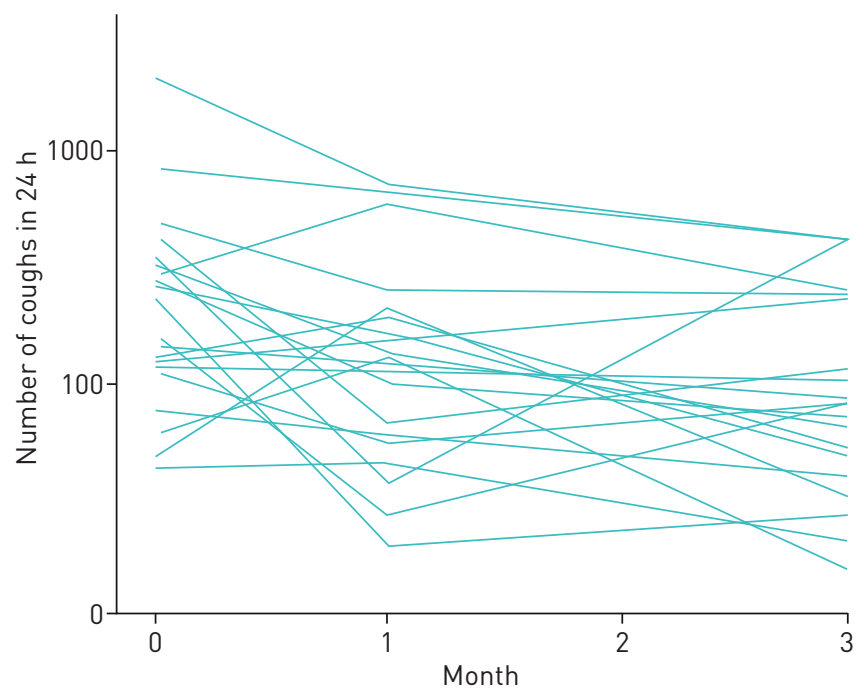

FIGURE 2 Number of coughs in $24 \mathrm{~h}$. Individual subject data showing 24-h cough counts at baseline and during treatment with azithromycin for 1 month and 3 months.

\section{Discussion}

In this noncontrolled open-label trial in people with sarcoidosis who reported a chronic cough, 3 months of treatment with azithromycin led to improvements in a range of cough metrics. The results support an RCT of azithromycin as a treatment for sarcoidosis cough.

Current concepts link chronic cough with hypersensitivity of airway nerves or their central connections [9]. In sarcoidosis, SinHa et al. [2] reported that only cough reflex sensitivity was associated with objective cough frequency, and there was no association with lung function, number of organs involved, chest radiography stage or serum angiotensin-converting enzyme. Cough is an important patient-reported trial end-point in sarcoidosis as it is a distressing and frequently disabling symptom for patients [2], and it may change more quickly and sensitively in response to treatment than lung function tests or radiology.

An appealing strategy is to repurpose existing drugs that are safe for long-term use. Azithromycin has an acceptable tolerability profile when used for long-term treatment of chronic airways diseases [4]. Macrolide therapy has the potential to modulate cough through antibacterial or immunomodulatory effects or promoting oesophageal motility through motilin agonism. Azithromycin is preferable to other macrolide antibiotics because of its safety data for long-term use, once-daily administration and minimal inhibition of liver CYP3A enzymes.

More than half of the patients with sarcoidosis cough treated with azithromycin reported clinically meaningful improvements in cough-related quality of life as indicated by a $\geqslant 1.3$-point increase in LCQ score [7], and more than three quarters had clinically meaningful improvements sarcoidosis-related quality of life measured using the KSQ_GH score. Correlations estimated that $41 \%$ of the increase in LCQ and $34 \%$ of the increase in KSQ_GH could be attributed to reduction in the number of measured coughs. The KSQ lung module (alone as KSQ_lung or combined with general health as KSQ_lung_GH) was less responsive to changes in cough count, and only one of the six lung-related questions pertains to cough. Although cough counting has the perceived benefit of being an objective end-point, our findings confirm that it correlates poorly with patient-reported outcomes. This will be an important consideration when designing a future RCT that focuses on improving symptoms.

The present study did not include a placebo arm, and a placebo response could explain some of the observed reduction in cough metrics. A modest but significant placebo response in subjective end-points has been observed in a meta-analysis of clinical trials of inhaled corticosteroids for chronic cough [10]. In sarcoidosis cough, Du BoIs et al.'s [11] study of inhaled fluticasone reported that in the placebo group, four-point cough scores fell by $17 \%$ at $1-3$ months and $40 \%$ at $4-6$ months. The mean increase in LCQ in the present study (3.47 points) following treatment with azithromycin compares favourably with changes in LCQ in published trials of azithromycin for chronic cough. In COPD patients with chronic cough, LCQ increased by mean 2.2 points with thrice-weekly azithromycin $250 \mathrm{mg}$ for 12 weeks and by 0.9 points with placebo [12]. In treatment-resistant chronic cough, LCQ increased by mean 2.4 points with thrice-weekly azithromycin $250 \mathrm{mg}$ for 8 weeks and by 0.7 points with placebo [13]. In the present study, the number of recorded coughs reduced by about half after 3 months of azithromycin therapy. Objective measurement of 

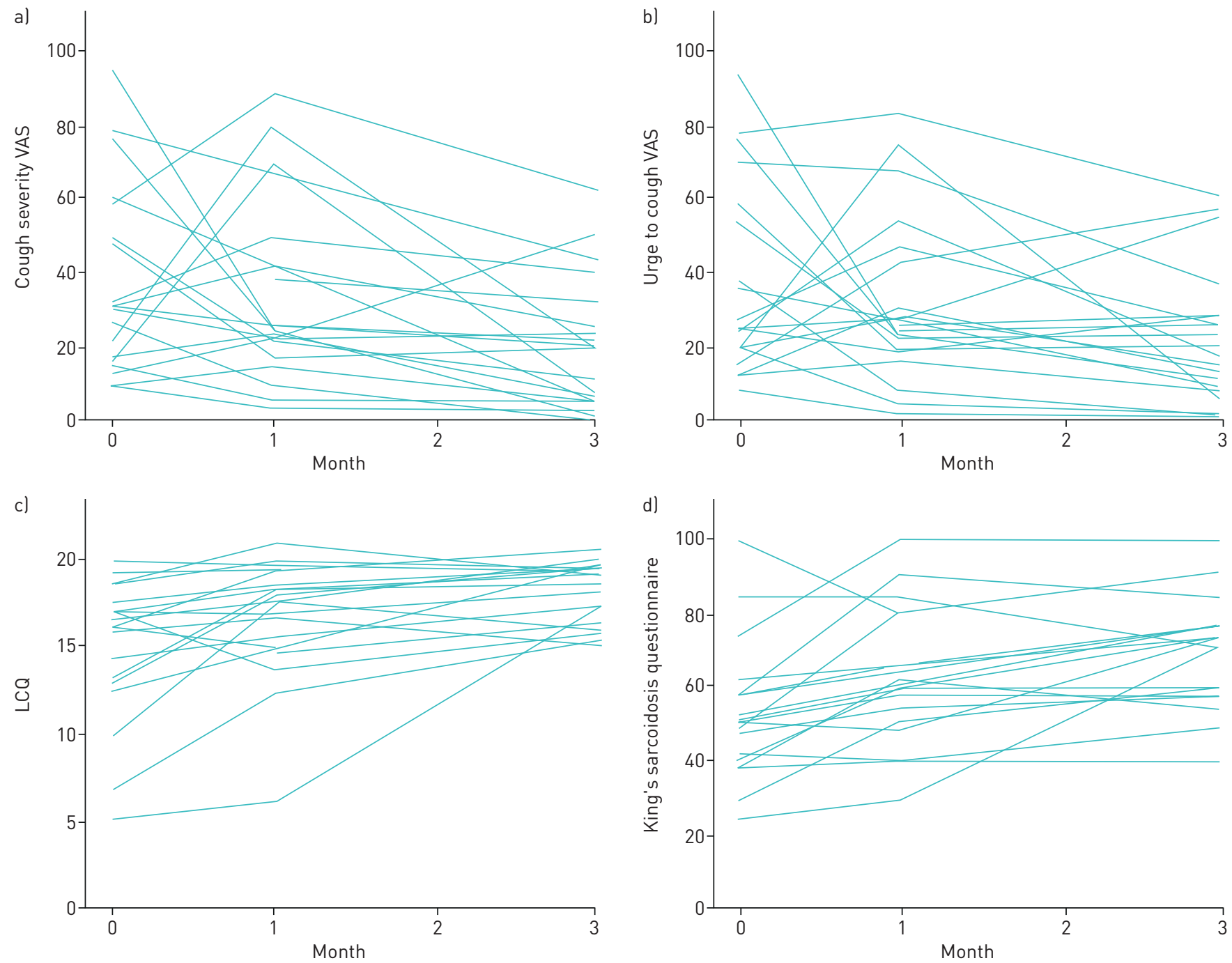

FIGURE 3 Patient-reported outcomes. Individual subject data showing: a) cough severity visual analogue scale (VAS); b) urge to cough VAS; c) Leicester cough questionnaire (LCQ) scores; and d) King's sarcoidosis questionnaire (KSQ) general health scores at baseline and during treatment with azithromycin for 1 month and 3 months. VAS are $100 \mathrm{~mm}$ scales with lower numbers indicating lower cough severity and urge to cough. Higher scores on LCQ and KSQ indicate better cough- and sarcoidosis-related quality of life respectively.

cough counting should be more resistant to placebo effect than subjective symptom scores [10]. There are no data in sarcoidosis specifically, but in a study of idiopathic pulmonary fibrosis (IPF) cough DuTTA et al. [14] reported that the mean $24 \mathrm{~h}$ cough frequency in the placebo group fell by $8.8 \%$ ( 8.3 from 9.1 ) over the 90-day treatment period. BIRRING et al. [15] reported stable 24-h cough counts at day 14 in the placebo group in their study of inhaled PA101 in IPF cough, albeit in patients with more severe cough at baseline compared to our sarcoidosis cohort. Unlike IPF, sarcoidosis may naturally remit which accounts for many transitory successes claimed at one time or another for a variety of therapeutic interventions [16]. We deliberately chose to study patients with chronic stable pulmonary sarcoidosis (median 3 years since diagnosis) to minimise risk of confounding by natural disease resolution and regression to the mean. We believe that the magnitude of objectively measured cough reduction with azithromycin is greater than expected for a placebo response alone.

Consideration should be given to inclusion criteria for a future RCT of azithromycin for sarcoidosis cough. We selected patients with self-reported chronic cough, and cough frequency in our cohort (median 10 coughs $\mathrm{h}^{-1}$ ) was somewhat lower than trials which have used cough severity VAS $\geqslant 40 \mathrm{~mm}$ as an arbitrary inclusion criterion (typically around 20 coughs $\cdot \mathrm{h}^{-1}$ ). A baseline cough count of $\geqslant 15$ coughs $\cdot \mathrm{h}^{-1}$ has been used for trials in IPF cough [15]. The rationale is that selecting participants who have more severe cough will maximise the chance of detecting a meaningful benefit of therapy and optimise clinical trial efficiency. In our sarcoidosis trial cohort, subjects with baseline cough severity VAS $\geqslant 40 \mathrm{~mm}$ had 


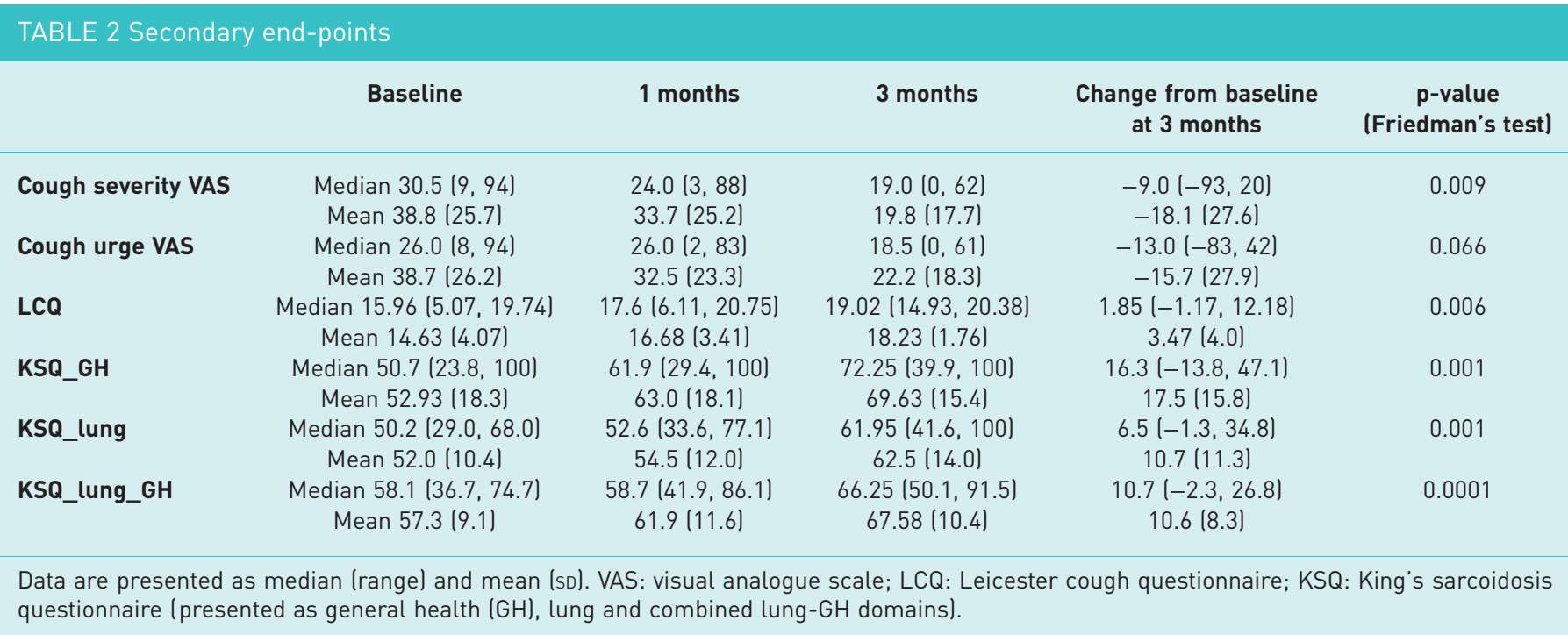

significantly more coughs in $24 \mathrm{~h}$ and a trend towards larger reduction in cough counts in response to treatment, although the latter did not reach statistical significance $(\mathrm{p}=0.057)$. On the other hand, only 8 of $21(38 \%)$ or our trial participants had a baseline cough severity VAS $\geqslant 40 \mathrm{~mm}$, which would restrict recruitment to a RCT compared with our broad inclusion of self-reported cough. It can be argued that for a treatment designed primarily to improve cough, patient-reported outcomes such as quality of life scores are more important trial end-points than counting coughs.

Azithromycin was well tolerated. The potential for macrolides to lengthen the QTc interval on ECG has not translated into risk of serious cardiac adverse events in several large trials of long-term therapy in patients with chronic airways disease $[4,17]$. The possibility of interaction with asymptomatic cardiac sarcoidosis needs to be considered when designing a future trial of azithromycin for sarcoidosis cough. The prevalence of occult cardiac involvement in sarcoidosis has been reported to be up to $25 \%$ as assessed using magnetic resonance imaging [18]. However, whether the presence of incidental cardiac imaging abnormalities translates into increased arrhythmia risk that could be exacerbated by macrolide therapy is unknown. A pragmatic approach is to exclude subjects with a history of serious cardiac arrhythmias or abnormal QTc prolongation on ECG at screening, and to perform serial ECG monitoring.

Both immunomodulatory and antibacterial properties of macrolides would be plausibly beneficial in sarcoidosis, but we do not know whether long-term macrolide therapy could ameliorate granulomatous inflammation or reduce sarcoidosis progression. Improvements in sarcoidosis end-points have been reported using antibiotic combinations that included azithromycin $[19,20]$. In the present exploratory study, we were not aiming to demonstrate an effect on disease progression in patients taking azithromycin because of the sample size limitation of our single-centre study and the imprecision of physiological end-points such as change in forced vital capacity. A large RCT would be needed to study this.

Acknowledgements: The LCQ and KSQ were developed by Surinder Birring and colleagues. We are grateful to Prof. Birring for advice on analysing the KSQ.

Conflict of interest: S.D. Fraser reports grants from SarcoidosisUK during the conduct of the study. S. Thackray-Nocera has nothing to disclose. M. Shepherd has nothing to disclose. R. Flockton has nothing to disclose. C. Wright has nothing to disclose. W. Sheedy has nothing to disclose. K. Brindle has nothing to disclose. A.H. Morice has nothing to disclose. P.M. Kaye reports grants from SarcoidosisUK during the conduct of the study. M.G. Crooks reports grants from SarcoidosisUK during the conduct of the study. S.P. Hart reports grants from SarcoidosisUK during the conduct of the study.

Support statement: This work was funded by a research grant from SarcoidosisUK. P.M. Kaye is supported by a Wellcome Trust Senior Investigator Award (WT104726). Funding information for this article has been deposited with the Crossref Funder Registry.

\section{References}

1 Harrison NK. Cough, sarcoidosis and idiopathic pulmonary fibrosis: raw nerves and bad vibrations. Cough 2013; 9: 9-9.

2 Sinha A, Lee KK, Rafferty GF, et al. Predictors of objective cough frequency in pulmonary sarcoidosis. Eur Respir J 2016; 47: 1461-1471. 
3 Morice AH, Millqvist E, Bieksiene K, et al. ERS guidelines on the diagnosis and treatment of chronic cough in adults and children. Eur Respir J 2020; 55: 1901136.

4 Smith D, Du Rand I, Addy CL, et al. British Thoracic Society guideline for the use of long-term macrolides in adults with respiratory disease. Thorax 2020; 75: 370-404.

5 Barry SJ, Dane AD, Morice AH, et al. The automatic recognition and counting of cough. Cough 2006; 2: 8.

6 Sinha A, Bajwah S, Gosker H, et al. A comparison of two scoring methods for the King's sarcoidosis questionnaire. Eur Respir J 2015; 46: Suppl. 59, PA832.

7 Raj AA, Pavord DI, Birring SS. Clinical cough IV: what is the minimal important difference for the Leicester Cough Questionnaire? Handb Exp Pharmacol 2009: 187: 311-320.

8 Patel AS, Spinou A, Keir G, et al. Assessing sarcoidosis: the King's sarcoidosis questionnaire and the minimal important difference. Eur Respir J 2012; 40: Suppl. 56, P699.

9 Morice AH, Millqvist E, Belvisi MG, et al. Expert opinion on the cough hypersensitivity syndrome in respiratory medicine. Eur Respir J 2014; 44: 1132-1148.

10 Lee SE, Lee JH, Kim HJ, et al. Inhaled corticosteroids and placebo treatment effects in adult patients with cough: a systematic review and meta-analysis. Allergy Asthma Immunol Res 2019; 11: 856-870.

11 du Bois RM, Greenhalgh PM, Southcott AM, et al. Randomized trial of inhaled fluticasone propionate in chronic stable pulmonary sarcoidosis: a pilot study. Eur Respir J 1999; 13: 1345-1350.

12 Berkhof FF, Doornewaard-ten Hertog NE, Uil SM, et al. Azithromycin and cough-specific health status in patients with chronic obstructive pulmonary disease and chronic cough: a randomised controlled trial. Respir Res 2013; 14 125.

13 Hodgson D, Anderson J, Reynolds C, et al. The effects of azithromycin in treatment-resistant cough: a randomized, double-blind, placebo-controlled trial. Chest 2016; 149: 1052-1060.

14 Dutta P, Funston W, Mossop H, et al. Randomised, double-blind, placebo-controlled pilot trial of omeprazole in idiopathic pulmonary fibrosis. Thorax 2019; 74: 346-353.

15 Birring SS, Wijsenbeek MS, Agrawal S, et al. A novel formulation of inhaled sodium cromoglicate (PA101) in idiopathic pulmonary fibrosis and chronic cough: a randomised, double-blind, proof-of-concept, phase 2 trial. Lancet Respir Med 2017; 5: 806-815.

16 Gibson GJ. Sarcoidosis: old and new treatments. Thorax 2001; 56: 336-339.

17 Albert RK, Schuller JL. Macrolide antibiotics and the risk of cardiac arrhythmias. Am J Respir Crit Care Med 2014; 189: $1173-1180$.

18 Martusewicz-Boros MM, Boros PW, Wiatr E, et al. Prevalence of cardiac sarcoidosis in white population: a case-control study: proposal for a novel risk index based on commonly available tests. Medicine 2016; 95 : e4518.

19 Drake WP, Oswald-Richter K, Richmond BW, et al. Oral antimycobacterial therapy in chronic cutaneous sarcoidosis: a randomized, single-masked, placebo-controlled study. JAMA Dermatol 2013; 149: 1040-1049.

20 Drake WP, Richmond BW, Oswald-Richter K, et al. Effects of broad-spectrum antimycobacterial therapy on chronic pulmonary sarcoidosis. Sarcoidosis Vasc Diffuse Lung Dis 2013; 30: 201-211. 